Alana Soares Brandão Barreto'

Marina Ferreira de Mederos Mendes ${ }^{2}$

Luiz Claudio Santos Thuler ${ }^{3}$

\section{Artigo Original}

Palavras-chave

Avaliação em saúde Programas de rastreamento Cobertura de serviços de saúde Neoplasias da mama Fidelidade a diretrizes

Keywords

Health evaluation

Mass screening

Health services coverage

Breast neoplasms

Guideline adherence

\title{
Avaliação de uma estratégia para ampliar a adesão ao rastreamento do câncer de mama no Nordeste brasileiro
}

\author{
Evaluation of a strategy adopted to expand adherence to breast \\ cancer screening in Brazilian Northeast
}

\section{Resumo}

OBJETIVO: Avaliar as ações do Programa "Um Beijo Pela Vida", desenvolvido em um município do Nordeste brasileiro visando ampliar a adesão ao rastreamento do câncer de mama em mulheres cadastradas pela Estratégia Saúde da Família. MÉTODOS: Utilizou-se abordagem quantitativa para avaliar a cobertura das ações de rastreamento nas mulheres com 40 anos de idade ou mais. Foi realizada busca ativa das mulheres por agentes comunitários de saúde das nove Equipes de Saúde da Família do município. Os percentuais das mulheres submetidas ao exame clínico das mamas e à mamografia; de exames na faixa etária recomendada pelo Programa; da classificação das lesões mamográficas, de acordo com o BI-RADS ${ }^{\circledR}$; das mulheres que receberam encaminhamento para diagnóstico e tratamento e o número de casos de câncer de mama detectados foram colhidos por meio de um questionário estruturado, analisados pelo programa EPI-INFO ${ }^{\mathrm{TM}}$ e confrontados a padrões previamente fixados. RESULTADOS: Foram incluídas 3.608 mulheres, correspondendo a $68,4 \%$ da população-alvo inscrita na Estratégia Saúde da Família. As taxas de cobertura do exame clínico das mamas para mulheres de 40 a 49 anos e da mamografia para mulheres de 50 a 69 anos foram de 58,9 e 56,7\%, respectivamente. As mulheres com lesões mamográficas altamente suspeitas foram submetidas à punção por agulha fina ou por agulha grossa (100\%). Seis casos novos de câncer foram detectados. Foram cumpridos $80 \%$ dos padrões analisados. CONCLUSÕES: A avaliação das ações do Programa aponta para sua adequação, considerando-se o grau de cumprimento das exigências previamente definidas.

\section{Abstract}

PURPOSE: To evaluate the actions of the "Um Beijo Pela Vida" Program developed in a Brazilian Northeast city to increase adherence to breast cancer screening by women registered by the Brazilian Family Health Strategy for breast cancer screening. METHODS: A quantitative approach was used to evaluate the coverage of screening actions for aged 40 years-old or more. Community workers from the nine Family Health Teams of the town carried out an active search. The percentage of eligible women who were screened for breast cancer by clinical breast examination or mammography, mammogram classification according to BI-RADS ${ }^{\circledR}$, women screened who were referred for further testing and treatment, and the number of breast cancers detected were collected by means of a structured questionnaire, analyzed with the EPI-INFO ${ }^{T M}$ software and compared to previously defined patterns. RESULTS: 3,608 women were included, corresponding to $68.4 \%$ of the target population registered in the Brazilian Family Health Strategy. Coverage rates of clinical breast examination for women aged 40 to 49 years-old and of mammograms for women aged 50 to 69 years-old were 58.9 and $56.7 \%$, respectively. All women with highly suspicious mammographic lesions were submitted to fine needle aspiration or core biopsy (100\%). Six new cases of cancer were detected and $80 \%$ of the standards established for this evaluation were carried out. CONCLUSIONS: The evaluation of the actions of the Program suggests its adequacy considering the degree of fulfillment of the previously defined requirements.

Correspondêncio

Marina Ferreira de Medeiros Mendes Grupo de Estudos de Gestão e Avaliação em Saúde - Instituto de Medicina Integral Professor Fernando Figueira. Rua dos Coelhos, 300 - Boa Vista (EP: 50070-550 Recife (PE), Brasil

Recebido

$11 / 08 / 2011$

Aceito com modificacoūes $02 / 01 / 2012$
Trabalho realizado na Secretaria de Estado da Saúde da Paraíba - Monteiro (PB), Brasil.

1 Instituto de Medicina Integral Professor Fernando Figueira - GEAS/IMIP - Recife (PE), Brasil; Coordenadoria de Planejamento e Controle dos Serviços de Saúde - Monteiro (RN), Brasil

${ }^{2}$ Grupo de Estudos de Gestão e Avaliação em Saúde do Instituto de Medicina Integral Professor Fernando Figueira - IMIP - Recife (PE), Brasil.

3 Instituto Nacional de Câncer - INCA; Universidade Federal do Estado do Rio de Janeiro - UNIRIO - Rio de Janeiro (RJ), Brasil. Conflito de interesses: não há. 


\section{Introdução}

O câncer de mama representa, no mundo, a neoplasia maligna mais frequentemente diagnosticada em mulheres. As estatísticas indicam o aumento de sua incidência tanto nos países desenvolvidos quanto nos em desenvolvimento. No Brasil, o câncer de mama é a principal causa de morte por câncer entre mulheres. Em 2012, a estimativa da doença é de 52.680 novos casos diagnosticados e com risco estimado de 52,5 para 100.000 mulheres ${ }^{1}$. No Estado da Paraíba as taxas de mortalidade por câncer de mama ajustadas por idade vêm crescendo nos últimos anos, passando de 2,8 óbitos por 100.000 mulheres em 1990 para 10,0 óbitos por 100.000, em 2009, o que equivale a uma variação percentual relativa de $257 \%$ em 20 anos $^{2}$.

Soma-se às crescentes taxas de incidência e mortalidade a constatação de que, no Brasil, o diagnóstico da doença ocorre quando a mesma já se encontra em nos estádios avançados. Em estudo realizado no Rio de Janeiro, o câncer de mama foi diagnosticado em fase avançada (estádios clínicos II, III e IV) em $51 \%$ dos casos analisados; entretanto, apenas 19,2\% dos casos assintomáticos, em que a lesão foi detectada por mamografia, apresentavam-se em estádio avançado ${ }^{3}$. Uma síntese de nove estudos brasileiros baseados em séries de casos hospitalares ou registros de câncer de base populacional, realizados entre 1993 e 2007 , mostrou que 45,4\% (valor mediano) dos 3.352 casos de câncer de mama estudados encontravam-se em estádio avançado (III ou IV) no momento do diagnóstico ${ }^{4}$. Há duas décadas, a situação era ainda mais crítica, quando 60 a $70 \%$ das mulheres eram diagnosticadas tardiamente ${ }^{5}$, as possibilidades de cura eram menores e a retirada total da mama se apresentava, muitas vezes, como a única opção de tratamento.

Para enfrentamento do câncer de mama no país, o Ministério da Saúde (MS), por meio do Instituto Nacional do Câncer (INCA), desenvolveu o Programa "Viva Mulher", que tem como objetivo reduzir a mortalidade e as repercussões físicas, psíquicas e sociais do câncer de mama na mulher brasileira, por meio da oferta de serviços para detecção do câncer em estágios iniciais, para tratamento e reabilitação. O INCA recomenda, para as mulheres com idades entre 50 a 69 anos, rastreamento por mamografia, com o máximo de dois anos entre os exames. Já o rastreamento por meio do exame clínico das mamas (ECM) deve ser oferecido anualmente para todas as mulheres a partir dos 40 anos. Mamografia e ECM anuais estão indicados a partir dos 35 anos de idade para as mulheres pertencentes a grupos populacionais com risco elevado de desenvolver câncer de mama. O INCA desta$\mathrm{ca}$, ainda, que o exame das mamas realizado pela própria mulher (autoexame) não substitui o físico realizado pelo profissional de saúde, treinado para tal atividade, e que, embora não exista evidência científica de seu impacto sobre a mortalidade pelo câncer de mama, seu estímulo deve fazer parte das ações de educação para a saúde que contemplem o conhecimento do corpo ${ }^{6}$.

Um estudo longitudinal, tendo por base $460 \mathrm{mu}-$ lheres atendidas em serviços de saúde públicos e privados do município de Taubaté, São Paulo, mostrou que 68\% delas referiam rastreamento mamográfico nos dois anos anteriores à pesquisa, sendo maior a proporção $(84,2 \%)$ entre aquelas que tinham cobertura de planos de saúde privados. Houve maior adesão (repetição da mamografia em intervalos máximos de 24 meses) em serviços privados, em mulheres na menopausa, com consultas ginecológicas ou com o mastologista regulares, com histórico de coleta de citologia oncótica e com rastreamento mamográfico prévio. A escolaridade, o estado marital, a ocupação profissional, a renda familiar, a presença de doenças mamárias e os antecedentes familiares de câncer de mama não apresentaram associação com a adesão ao rastreamento mamográfico ${ }^{7}$. Outro estudo, envolvendo 3.749 mulheres carentes atendidas no Núcleo Mama Porto Alegre, revelou que ter risco genético elevado, maior escolaridade, história de uso de contraceptivo oral, até dois filhos e ser ex-fumante estão associados de maneira independente à maior adesão (intervalo entre exames menor ou igual a 18 meses) à mamografias .

Com a finalidade de aumentar a adesão à mamografia e ao exame de Papanicolau de mulheres de 19 cidades da região de Barretos, em São Paulo, ao utilizar-se a distribuição de volantes e panfletos, chamadas por meio de rádio e carros de som e visitas domiciliares por agentes comunitários de saúde (ACS), mostrou-se ser esta última a estratégia mais efetiva ${ }^{9}$. Em outro estudo envolvendo uma população predominantemente de mulheres afroamericanas, observou-se que o uso de informação dirigida às pacientes, lembretes, reuniões, eventos comunitários, programas em igrejas e meios de comunicação aumentaram significativamente a adesão à mamografia de 31 para $56 \%{ }^{10}$.

Neste contexto, este estudo teve por objetivo avaliar as ações do Programa "Um Beijo Pela Vida", desenvolvido em um município do Nordeste brasileiro.

\section{Métodos}

Foi realizado um estudo de avaliação da cobertura das ações de rastreamento do câncer de mama no Município de Monteiro, localizado na Região do Cariri Ocidental, extremo Sul do Estado da Paraíba, distante $320 \mathrm{~km}$ de João Pessoa. Monteiro possui gestão plena de sistema de saúde, população de 28.230 habitantes, entre os quais 13.874 são do sexo masculino e 14.356 do feminino, sendo que 3.883 das mulheres têm idade superior ou igual a $40 \operatorname{anos}^{2}$. O município é dotado de nove unidades da 
Estratégia de Saúde da Família (ESF), com cobertura de $100 \%$ da população, e de um serviço de média complexidade estruturado para a realização dos procedimentos clínicos, cirúrgicos e com finalidade diagnóstica. Os casos de câncer diagnosticados são tratados em centros de referência localizados fora do município. A intensificação das ações de controle do câncer de mama ocorreu por meio de uma estratégia de saúde pública, chamada "Um Beijo Pela Vida", que teve como objetivo aumentar a adesão das mulheres às ações de detecção precoce do câncer de mama. Desenvolvido pela Secretaria de Estado da Saúde, com o apoio do Instituto Avon e da Cunhã Coletivo Feminista, a ação teve como bases técnicas a publicação do "Controle do Câncer de Mama: Documento de Consenso" ", dos Parâmetros Técnicos para Programação de Ações de Detecção Precoce do Câncer de $\mathrm{Mama}^{11}$ e do Pacto pela Vida ${ }^{12}$. Este preconiza que os municípios devem comprometer-se a ampliar para $60 \%$ a cobertura de rastreamento mamográfico e garantir punção diagnóstica em $100 \%$ dos casos, nos quais houver necessidade.

Foi elaborado um fluxograma ${ }^{13}$ das ações a serem desenvolvidas pela Secretaria Municipal de Saúde, de acordo com as recomendações do MS. Para a concretização dessas ações, ACS foram capacitados para captar as mulheres a serem submetidas ao ECM e à mamografia na unidade de saúde da família e orientá-las sobre a adoção de hábitos saudáveis na vida. Foram também capacitados os médicos e enfermeiros das unidades a fim de que realizassem os exames clínicos das mamas e encaminhassem adequadamente os casos ao serviço de referência. O Programa também disponibilizou equipamentos de informática, tais como computador e impressora, para os registros dos dados na unidade secundária, e materiais didáticos produzidos exclusivamente para o Programa a serem utilizados na abordagem das mulheres, tais como folders, cartazes e livretos para agentes de saúde. Também foi utilizado um modelo de silicone para ensinar a técnica de palpação das mamas. Além disso, foram realizadas reuniões de planejamento, redimensionamento da oferta de serviços e identificação de referências para tratamento. Baseando-se nas recomendações do MS, foi possível construir o modelo lógico do Programa.

Utilizando um instrumento de coleta de dados estruturado, construído especialmente para esta pesquisa, ocorreram visitas domiciliares, tendo como alvo mulheres na faixa etária de 40 a 69 anos, residentes no município de Monteiro. ACS das equipes de saúde da família, especialmente treinados para este fim, entrevistaram as mulheres entre maio e novembro de 2008. Os dados foram inseridos em um banco de dados criado para este fim, e informações referentes aos exames complementares feitos entre 2001 a 2007 foram colhidas do banco de dados do Centro de Atenção à Saúde da Mulher, que é a única referência da região para os procedimentos diagnósticos do câncer de mama. Os laudos mamográficos foram emitidos de acordo com o sistema BI-RADS ${ }^{\circledR}$ (Breast Imaging Reporting and Data System $)^{13}$, sendo os laudos classificados em seis categorias: 1 - sem achados, 2 - achados benignos, 3 achados provavelmente benignos, 4 - achados suspeitos, 5 - achados altamente suspeitos e 0 - achados que necessitam de avaliação adicional. Outras informações relevantes foram colhidas da base de dados existente nos serviços de atenção básica, nas unidades de saúde da família e nos serviços de referência de média e alta complexidade para onde as mulheres foram encaminhadas.

Para a análise dos dados, utilizou-se o programa EPIINFO $^{\text {TM }}$. Foram descritas as características demográficas e epidemiológicas das mulheres incluídas no estudo. Foram ainda analisados os seguintes indicadores de qualidade do Programa: percentuais de mulheres que foram submetidas ao ECM e à mamografia (cobertura) e de exames na faixa etária prioritária do Programa; classificação das lesões mamográficas de acordo com o BI-RADS ${ }^{\circledR}$. Ainda, os percentuais de mulheres, que receberam encaminhamento para esclarecimento diagnóstico em serviços de referência secundária e para tratamento em serviços de referência terciária, foram estudados.

Para cada pergunta avaliativa foram estabelecidos critérios específicos a fim de se fazer uma apreciação das respostas obtidas. Uma matriz de julgamento foi construída, na qual avaliou-se o grau de cumprimento dos padrões estabelecidos, atribuindo-se os seguintes juízos de valor: 75 a $100 \%$-adequado; 50 a $75 \%$ - parcialmente adequado; 25 a $50 \%$ - não-adequado e 0 a $25 \%$ - crítico.

A pesquisa foi conduzida dentro dos padrões regulamentados pela portaria 196/96 e foi aprovada pela Comissão de Ética da Secretaria de Estado da Saúde da Paraíba em 26 de fevereiro de 2008, recebendo o registro 173.272 .

\section{Resultados}

De um universo de 5.278 mulheres inscritas nas nove equipes da ESF do Município de Monteiro, 3.608 $(68,4 \%)$ foram entrevistadas em seus domicílios. Em apenas uma equipe, situada em zona urbana, obteve-se percentual de inclusão inferior a $50 \%$. As características epidemiológicas e demográficas das mulheres incluídas neste estudo estão descritas na Tabela 1 . A média de idade foi de 58,2 anos, sendo que cada mulher teve, em média, 5,8 gestações. Observou-se ainda que 1,3 e 2,3\% das mulheres apresentavam história de mãe ou irmã com câncer de mama, respectivamente. As demais características clínicas e referentes à utilização dos serviços estão dispostas na Tabela 2. Vale ressaltar que $70,8 \%$ relataram ter sido submetidas ao exame citopatológico do colo do útero pelo 
Tabela 1. Características epidemiológicas e demográficas das mulheres inscritas nas equipes da saúde da família incluídas no estudo

\begin{tabular}{|c|c|c|}
\hline Variável & Média (DP) & $\begin{array}{l}\text { Mínimo- } \\
\text { Máximo }\end{array}$ \\
\hline Idade em anos ( $n=3.608)$ & $58,2(13,3)$ & $40-103$ \\
\hline Idade em anos da primeira menstruação ( $n=3.528)$ & $13,7(1,6)$ & $8-19$ \\
\hline Idade em anos da menopausa ( $n=2.400)$ & $46,5(6,0)$ & $20-70$ \\
\hline Número de gestações ( $n=3.605)$ & $5,8(4,9)$ & $0-30$ \\
\hline Número de abortos ( $n=3.604$ ) & $0,7(1,3)$ & $0-12$ \\
\hline Número de cesarianas ( $n=3.604$ ) & $0,2(0,6)$ & $0-12$ \\
\hline Idade da primeira gestação, em anos ( $n=3.605$ ) & $18,9(8,8)$ & $0-49$ \\
\hline Tempo de amamentação, em meses ( $n=3.589)$ & $30,0(52,5)$ & $0-240$ \\
\hline
\end{tabular}

menos uma vez na vida, e que 58,9 e 49,0\% tinham sido submetidas a ECM ou mamografia, respectivamente.

Observou-se que $62,1 \%$ das mulheres encontravam-se cobertas pelas estratégias de detecção precoce do câncer de mama. Esse percentual foi maior entre mulheres com idades entre 40 e 49 anos (72,2\%, sendo 18,4\% por ECM, 3,5\% por mamografia e $50,3 \%$ por ambos) e menor entre aquelas com 70 anos ou mais $(34,2 \%$, sendo $8,0 \%$ por ECM, $3,1 \%$ por mamografia e $23,1 \%$ por ambos). Na faixa etária de 50 a 69 anos, a cobertura foi de $68,7 \%$, sendo $11,9 \%$ por ECM, $53,7 \%$ por mamografia e $3,1 \%$ por ambos. Além disso, em $73,6 \%$ dos casos, as mulheres foram submetidas aos dois exames (ECM e mamografia), não havendo variação importante conforme a faixa etária. Das mulheres submetidas ao ECM, 88,2\% encontravam-se na faixa etária indicada pelo Programa (40 a 69 anos), enquanto entre aquelas submetidas à mamografia, 51,6\% encontravam-se na faixa etária recomendada pelo MS (50 a 69 anos).

A análise do banco de dados do Centro de Atenção à Saúde da Mulher permitiu identificar que, entre aquelas que referiam terem sido submetidas à mamografia, os laudos foram localizados em 55,7\% dos casos. Foram observados os seguintes resultados: 81 casos BIRADS ${ }^{\circledR} 0$ $(7,6 \%) ; 6$ BIRADS $^{\circledR} 1(58,9 \%) ; 3$ BIRADS $^{\circledR} 2(30,5 \%)$ e 17 BIRADS $^{\circledR} 3(1,6 \%)$. Nas categorias suspeitas de malignidade, foram encontrados 15 casos: $10(0,9 \%)$ pacientes com BIRADS ${ }^{\circledR} 4$ e $5(0,5 \%)$ com BIRADS ${ }^{\circledR} 5$. Dezessete mulheres (todos os casos de BIRADS ${ }^{\circledR} 4$ ou 5 , e dois casos com lesões palpáveis nos quais o BIRADS ${ }^{\circledR}$ foi 1 ou 2) foram submetidas à punção por agulha fina e/ou por agulha grossa (core biopsy). Uma paciente foi submetida aos dois procedimentos. Das seis pacientes encaminhadas para cirurgia, cinco apresentavam câncer de mama nos estádios II e III e uma apresentava carcinoma in situ. A taxa de detecção de câncer de mama foi de $0,34 \%$ (seis casos em 1.767 mamografias analisadas).

$\mathrm{Na}$ Tabela 3 está apresentado o percentual de mamografias consideradas altamente suspeitas, conforme o número de exames. A periodicidade do exame está associada a uma importante diminuição no percentual
Tabela 2. Características clínicas e utilização de serviços de saúde pelas mulheres entrevistadas

\begin{tabular}{|c|c|}
\hline Variável & $n(\%)$ \\
\hline História de uso de contraceptivo oral ( $n=1.445)$ & $530(36,7)$ \\
\hline História de uso de terapia de reposição hormonal ( $n=696$ ) & $288(41,4)$ \\
\hline História de cirurgia prévia (n=3.606) & $34(0,9)$ \\
\hline História de mãe com câncer de mama ( $n=3.608$ ) & $46(1,3)$ \\
\hline História de irmã com câncer de mama ( $n=3.608$ ) & $84(2,3)$ \\
\hline História de inflamação na mama ( $n=3.608$ ) & $3,7(1,0)$ \\
\hline História de nódulo na mama ( $n=3.608$ ) & $94(2,6)$ \\
\hline Exame citopatológico do colo do útero prévio $(n=2.662)$ & $2.553(95,9)$ \\
\hline Exame clínico das mamas ( $n=3.608)$ & $2.126(58,9)$ \\
\hline Mamografia $(n=3.608)$ & $1.767(49,0)$ \\
\hline Ultrassonografia mamária ( $n=3.608$ ) & $214(5,9)$ \\
\hline Punção por agulha fina (n=3.608) & $13(0,4)$ \\
\hline Punção por agulha grossa (core biopsy) ( $\mathrm{n}=3.608)$ & $5(0,1)$ \\
\hline
\end{tabular}

Tabela 3. Percentual de mamografias com lesões altamente suspeitas (BIRADS 4 e 5), conforme o ranqueamento do exame no Centro de Atenção à Saúde da Mulher (CASM)

\begin{tabular}{l|cc}
\hline $\begin{array}{l}\text { Ranqueamento do exame } \\
\text { no CASM }\end{array}$ & $\begin{array}{c}\text { Mamografias realizadas } \\
\text { no CASM }\end{array}$ & $\begin{array}{c}\text { BIRADS 4 E 5 } \\
(\%)\end{array}$ \\
\hline $1^{\circ}$ exame & 2.737 & 1,2 \\
$2^{\circ}$ exame & 1.219 & 0,7 \\
$3^{\circ}$ exame & 620 & 0,5 \\
$4^{\circ}$ exame ou mais & 425 & 0,5 \\
TOTAL & 5.001 & 0,9 \\
\hline
\end{tabular}

Fonte: CASM/Monteiro/Paraíba.

de exames suspeitos, o que já pode ser observado a partir da segunda mamografia.

Por fim, os dados coletados foram inseridos na matriz de julgamento do Programa, comparando-se o observado (coletado pela pesquisa) com o esperado (padrões estabelecidos). De acordo com os valores obtidos, o Programa pode ser considerado como adequado pela análise dos aspectos avaliados por esta pesquisa.

\section{Discussão}

Este estudo objetivou avaliar uma estratégia de saúde pública, desenvolvida com a finalidade de ampliar a adesão das mulheres às ações de rastreamento do câncer de mama no Município de Monteiro - Estado da Paraíba. A avaliação consistiu fundamentalmente em fazer um julgamento de valor sobre uma intervenção. Deve servir para direcionar ou redirecionar a execução de ações, atividades, programas e, por conseguinte, deve ser exercida por aqueles envolvidos no planejamento e na execução de tais ações ${ }^{14,15}$.

O presente estudo se propôs a conhecer a cobertura do ECM e da mamografia na população-alvo do programa de controle do câncer de mama em um município do interior da Paraíba. A avaliação de cobertura mede a proporção da 
população que se beneficia do programa ou da intervenção ${ }^{16}$ e responde à pergunta formulada por muitos gestores, no que se refere à magnitude da oferta das ações e do serviços, tal como: em que medida os recursos existentes são suficientes para atender à população-alvo.

No que diz respeito à cobertura das ações, neste estudo foi identificado que o Programa "Um Beijo Pela Vida" cobriu $68,4 \%$ da população feminina com mais de 40 anos de idade cadastrada na ESF. A taxa de participação aproximou-se daquela observada em programa semelhante desenvolvido em Mato Grosso do Sul, no qual houve adesão de $74,9 \%{ }^{17}$.

Neste estudo, entre as mulheres entrevistadas, observou-se que, na faixa etária entre 50 e 69 anos, 58,9\% haviam sido avaliadas com exame clínico das mamas, enquanto a cobertura do exame mamográfico foi de $56,8 \%$. Embora existam poucas informações da cobertura de rastreamento mamográfico em mulheres atendidas nos serviços públicos de saúde de cidades do interior do país, observou que $53,2 \%$ daquelas de 40 a 69 anos atendidas pela ESF no município de Dourados, no Mato Grosso do Sul, referiam ter sido submetidas à mamografia prévia ${ }^{18}$, valor semelhante ao descrito em Monteiro. Entretanto, em Taubaté, em São Paulo ${ }^{7}$, observou-se que 61,5\% das mulheres atendidas no Sistema Único de Saúde (SUS) referiam exame mamográfico prévio, contra $84,2 \%$ daquelas atendidas nos serviços privados.

Os valores de cobertura do ECM e do mamográfico, descritos em Monteiro, são superiores ao dobro daqueles encontrados pela Pesquisa Nacional por Amostra de Domicílio ${ }^{19}$, conduzida pelo Instituto Brasileiro de Geografia e Estatística (IBGE), que, na Paraíba, mostrou que $24,4 \%$ das mulheres com 40 anos ou mais haviam sido submetidas ao ECM realizado por médico ou enfermeiro nos 12 meses anteriores à data da entrevista. $27,8 \%$ das mulheres de 50 a 69 anos de idade haviam sido submetidas ao exame mamográfico nos dois anos anteriores à data da entrevista. Em relação a esta última comparação, em geral, espera-se que os percentuais de cobertura nas capitais sejam superiores aos descritos no interior dos estados, já que é onde concentra-se um maior número de profissionais e de serviços de saúde. Portanto, em Monteiro, foram obtidos valores muito próximos daqueles da Capital do Estado, reforçando a ideia de que a intervenção em um município do interior ampliou o acesso das mulheres ao ECM e à mamografia.

Neste estudo a taxa de indicação de biópsias foi de $1 \%$ entre as mulheres rastreadas por mamografia (17 em $1767)$, valor inferior ao encontrado em estudo levado a termo em Campinas ${ }^{20}$, baseado em 35.041 mamografias consecutivas $(1,7 \%)$. Em Campinas, a taxa de detecção de câncer de mama entre pacientes assintomáticas foi de $0,3 \%$, exatamente o mesmo encontrado no presente estudo. Taxas inferiores foram descritas em Mato Grosso do Sul (0,1 casos por 100 mulheres rastreadas), em que se empregou o ECM como estratégia de rastreamento ${ }^{17}$. $\mathrm{O}$ American College of Radiology ${ }^{21}$ considera aceitáveis as taxas de detecção que variem entre 0,2 e 1,0\%.

No que diz respeito à distribuição dos estádios clínicos, enquanto em Campinas ${ }^{20}$ foram identificados $93 \%$ dos cânceres nos estádios 0 e I, em Monteiro esse percentual foi de $20 \%$. Deve ser destacado, no entanto, que, no atual estudo, mais da metade das mulheres $(51,0 \%)$ nunca tinham sido submetidas à mamografia, diferentemente da população estudada em Campinas, na qual a taxa de exposição prévia à mamografia era superior a $90 \%$.

Outro aspecto que deve ser destacado é que foram observadas taxas de detecção de exames descritos como altamente suspeitos (BIRADS ${ }^{\circledR} 4$ e 5), diferenciadas conforme o ranqueamento do exame, variando de $1,2 \%$ quando se tratava do primeiro exame a valores próximos a $0,5 \%$, quando se tratava do terceiro exame ou superior. Esse decaimento nas taxas explica-se pela presença, na primeira rodada, de casos prevalentes (que vêm se desenvolvendo por algum tempo) e de casos incidentes (desenvolvidos recentemente); já as mamografias subsequentes geralmente detectam anomalias incidentes, desenvolvidas recentemente. A análise dos resultados das mamografias de 230.143 mulheres, com mais de 40 anos, inseridas no Programa Norte-americano de Detecção Precoce dos Cânceres de Mama e do Colo do Útero (CDC's National Breast and Cervical Cancer Early Detection Program $)^{22}$, mostrou que o percentual de exames altamente sugestivos de malignidade também caía pela metade comparando mulheres que faziam o exame pela primeira vez àquelas em exames subsequentes, passando de 5,5 para 2,7\%. Há que se considerar os diferentes riscos para câncer de mama nessas populações.

Além disso, a taxa de lesões altamente suspeitas encontrada no primeiro exame $(1,2 \%)$ aproxima-se daquela referente aos laudos de mamografias (mamografia unilateral - código 02.04.03.003-0 e bilateral para rastreamento código 02.04.03.018-8), que foram digitados no Sistema de Informação do Câncer de Mama (SISMAMA) e estão disponíveis no site do DATASUS ${ }^{2}$. Neste, observa-se que, para o Brasil, das 724.628 mamografias efetuadas no SUS entre janeiro e dezembro de 2009, em mulheres entre 40 e 69 anos de idade, $1,9 \%$ foram classificadas como altamente suspeitas (BIRADS ${ }^{\circledR} 4$ e 5 ).

Com base nas informações disponíveis e na análise da matriz de julgamento das ações do Programa "Um Beijo Pela Vida", conclui-se pela sua adequação no que diz respeito ao cumprimento dos padrões estabelecidos para esta avaliação. Entretanto, os percentuais de mulheres atendidas na ESF, que foram submetidas ao ECM (40 anos ou mais) e à mamografia (50 anos ou mais), mostraram-se aquém do desejado. 
1. Brasil. Ministério da Saúde. Instituto Nacional de Câncer [Internet]. Estimativa 2012: incidência de câncer no Brasil. Rio de Janeiro: INCA; 2011 [cited 2011 Dez 15]. Available from: http://www. inca.gov.br/estimativa/2012/estimativa20122111.pdf

2. Brasil. Ministério da Saúde. DATASUS [Internet]. Indicadores de mortalidade. Brasília (DF): Ministério da Saúde; 2010 [cited 2011 Dez 15]. Available from: http://tabnet.datasus.gov.br/cgi/tabcgi. exe?idb2010/c10.def

3. Rezende MCR, Koch HA, Figueiredo JA, Thuler LCS. Causas do retardo na confirmação diagnóstica de lesões mamárias em mulheres atendidas em um centro de referência do Sistema Único de Saúde no Rio de Janeiro. Rev Bras Ginecol Obstet. 2009;31(2):75-81.

4. Thuler LCS. Epidemiologia do câncer de mama. In: Chagas CR, Menke CH, Vieira RJS, Boff RA, organizadores. Tratado de mastologia da SBM. Rio de Janeiro: Revinter; 2010. p. 487-91.

5. Martins E, Freitas-Junior R, Curado MP, Freitas NMA, Oliveira JC, Silva CMB. Evolução temporal dos estádios do câncer de mama ao diagnóstico em um registro de base populacional no Brasil central. Rev Bras Ginecol Obstet. 2009;31 (5):219-23.

6. Brasil. Ministério da Saúde. Instituto Nacional de Câncer. Controle do câncer de mama: documento de consenso [Internet]. Rio de Janeiro: INCA; 2004 [cited 2010 Maio 4]. Available from: http:// www.inca.gov.br/publicacoes/Consensointegra.pdf

7. Marchi AA, Gurgel MSC. Adesão ao rastreamento mamográfico oportunístico em serviços de saúde públicos e privados. Rev Bras Ginecol Obstet. 2010;32(4):191-7.

8. Caleffi M, Ribeiro RA, Bedin AJ Jr., Viegas-Butzke JM, Baldisserotto $F D$, Skonieski GP, et al. Adherence to a breast cancer screening program and its predictors in underserved women in southern Brazil. Cancer Epidemiol Biomarkers Prev. 2010;19(10):2673-9.

9. Mauad EC, Nicolau SM, Moreira LF, Haikel RL Jr., Longatto-Filho A, Baracat EC. Adherence to cervical and breast cancer programs is crucial to improving screening performance. Rural Remote Health. 2009;9(3): 1241.

10. Paskett ED, Tatum CM, D'Agostino R Jr., Rushing J, Velez R, Michielutte $R$, et al. Community-based interventions to improve breast and cervical cancer screening: results of the Forsyth County Cancer Screening (FoCaS) Project. Cancer Epidemiol Biomarkers Prev. 1999;8(5):453-9.

11. Brasil. Ministério da Saúde. Secretaria de Atenção à Saúde. Instituto Nacional de Câncer. Coordenação de Prevenção e Vigilância. Parâmetros técnicos para programação de ações de detecção precoce do câncer da mama: recomendações para gestores estaduais e municipais. Rio de Janeiro: INCA; 2006.
12. Brasil. Ministério da Saúde. Portaria n. 399, de 22 de fevereiro de 2006. Divulga o Pacto pela Saúde 2006 - Consolidação do SUS e aprova as Diretrizes Operacionais do Referido. Diário Oficial da União, Brasília (DF); 2006. Seção 1, p. 43.

13. Souza EHR. Programa Viva Mulher e a detecção do câncer de mama no Cariri Ocidental da Paraíba [dissertação]. João Pessoa: Universidade Federal da Paraíba; 2007.

14. Champagne F, Contandriopoulos AP, Brousselle A, Hartz Z, Denis JL. L'évaluation dans le domaine de la santé: concepts et méthodes. In: Brousselle A, Champagne F, Contandriopoulos AP, Hartz Z. L'évaluation: concepts et méthodes. Montréal: Les Presses de I'Université de Montréal; 2009. p. 35-56.

15. Tanaka OY, Melo C. Reflexões sobre a avaliação em serviços de saúde e a adoção das abordagens qualitativa e quantitativa. In: Bosi MLM, Mercado-Martinez FJ, organizadores. Pesquisa qualitativa de serviços de saúde. Petrópolis: Vozes; 2004. p. 121-36.

16. Frias PG, Costa JMBS, Figueiró AC, Mendes MFM, Vidal SA. Atributos da qualidade em saúde. In: Samico I, Felisberto $E$, Figueiró AC, Frias PG, organizadores. Avaliação em saúde: bases conceituais e operacionais. Rio de Janeiro: Medbook; 2010. p. 43-6.

17. Thuler LC, Freitas HG. Evaluation of a community-based intervention to enhance breast cancer screening practices in Brazil. J Eval Clin Pract. 2008; 14(6): 1012-7.

18. Batiston AP. Detecção precoce do câncer de mama: conhecimento e prática de mulheres e profissionais da Estratégia de Saúde da Família em Dourados/MS. Rev Bras Ginecol Obstet. 2010;32(2):99.

19. Instituto Brasileiro de Geografia e Estatística. Pesquisa Nacional por Amostra de Domicílios. Um panorama da saúde no Brasil: acesso e utilização dos serviços, condições de saúde e fatores de risco e proteção à saúde - 2008. Rio de Janeiro: IBGE; 2010.

20. Camargo Júnior HSA, Camargo MMA, Teixeira SRC, Arruda MS, Azevedo J. Apresentação de resultados de um serviço de rastreamento mamográfico com ênfase na auditoria epidemiológica. Rev Bras Ginecol Obstet. 2009;31(10):508-12.

21. American College of Radiology (ACR). ACR BI-RADS ${ }^{\circledR}$-Mammography. ACR Breast Imaging Reporting and Data System Atlas. Reston: American College of Radiology; 2003.

22. May DS, Lee NC, Nadel MR, Henson RM, Miller DS. The National Breast and Cervical Cancer Early Detection Program: report on the first 4 years of mammography provided to medically underserved women. AJR Am J Roentgenol. 1998;170(1): 97-104 